\title{
On the Mutual Effect of Viscoplasticity and Interfacial Damage Progression in Interfacial Fracture of Lead-Free Solder Joints
}

\author{
MILAD MALEKI,${ }^{1}$ JOEL CUGNONI, ${ }^{1,2}$ and JOHN BOTSIS ${ }^{1}$ \\ 1.-Laboratoire de Mécanique Appliquée et d'Analyse de Fiabilité, Ecole Polytechnique Fédérale \\ de Lausanne (EPFL), CH-1015 Lausanne, Switzerland. 2.-e-mail: joel.cugnoni@epfl.ch
}

The main goal of this paper is to shed light on the effect of strain rate and viscoplastic deformation of bulk solder on the interfacial failure of lead-free solder joints. For this purpose, interfacial damage evolution and mode I fracture behavior of the joint were evaluated experimentally by performing stable fracture tests at different strain rates employing an optimized tapered double cantilever beam (TDCB) design. The viscoplastic behavior of the solder was characterized in shear, and the constitutive parameters related to the Anand model were determined. A rate-independent cohesive zone damage model was identified to best simulate the interfacial damage progression in the TDCB tests by developing a three-dimensional (3D) finite-element (FE) model and considering the viscoplastic response of the bulk solder. The influence of strain rate on the load capability and failure mode of the joint was clarified by analyzing the experimental and simulation results. It was shown how, at the lower strain rates, the normal stress generated at the interface is limited by the significant creep relaxation developed in the bulk solder and thus is not sufficiently high to initiate interfacial damage, whereas at higher rates, a large amount of the external energy is dissipated into interfacial damage development.

Key words: Lead-free solder, interfacial failure, viscoplastic deformation, Anand constitutive model, cohesive zone modeling

\section{INTRODUCTION}

As joining materials, solders provide electrical, thermal, and mechanical continuity in electronics assemblies. For several years, the eutectic composition of $\mathrm{Sn}-\mathrm{Pb}$ has been widely used as the main solder in the microelectronics industry for its advantageous properties. ${ }^{1}$ However, it is well established that lead is a poisonous material that can cause serious health problems in humans. This fact along with legislative directives for the suppression of lead in packaging systems gave rise to a large number of investigations on the development of substitutes for the classical lead solder alloy in the last two decades. ${ }^{1-3}$ Thanks to the works so far, use of lead-free solders has become a reality in consumer electronic products. However, the reliability of the lead-free solders is still a crucial issue,

(Received March 22, 2011; accepted July 11, 2011;

published online August 10, 2011) and in several cases the failure of the electronic assembly has been attributed to that of the solder joints. $^{2}$ Failure of the solder joint can mainly take place in two different modes: first, due to viscoplastic deformation accumulation and final failure in the bulk solder, and second, interfacial damage and crack growth causing fracture along the interface of the solder and the substrate. ${ }^{4-9}$

Several experimental and theoretical investigations on the creep properties and viscoplastic nature of lead-free solders have led to good insight into this subject. $^{4,5,10-13}$ However, comparison of the experimental results obtained in various studies shows considerable disagreement in several cases. Indeed, a review of identified constitutive parameters related to the hyperbolic sine model in different studies confirms the existence of wide scatter in the creep property database of the solders. ${ }^{12}$ Wiese and Wolter ${ }^{4}$ and Sidhu et al. ${ }^{12}$ discussed the critical role of the microstructures in the mechanical behavior of 
the solder joints. They attributed the scatter in the creep data to the differences in the microstructures of the solder specimens employed in various studies. Moreover, Frear and Vianco ${ }^{9}$ showed that development of interfacial damage during tension of the solder joints may influence the results of the tensile tests.

Previous studies indicated that the fracture behavior of the joint mainly depends on the strength of the solder alloy. ${ }^{9,14-16}$ While low-strength solders fail in the bulk by plastic deformation, the failure mode of high-strength solders depends on the thickness of the intermetallic layer. In the case of thin interfacial intermetallic compound (IMC), fracture may occur in the bulk solder or at the solder-IMC interface (IMC/solder delamination); otherwise it happens in the IMC layer (IMC cleavage). ${ }^{9,14-16}$ In addition to the solder strength and IMC thickness, some other factors such as the geometry of the joint, working temperature, microstructural evolution, roughness of the interface, and the amount of voids can affect the extent of the failure mode. ${ }^{5,15,17-19}$

The mechanics of the interfacial failure of solder joints were considered in several studies. ${ }^{14,20-27}$ Hayes et al. ${ }^{14}$ experimentally estimated the mode I fracture toughness of solder joints by performing high-strain-rate fracture tests on modified compact tension specimens. Kumar et al. ${ }^{24}$ went further and determined the mixed-mode fracture toughness of SAC387 at a wide range of strain rates in the framework of linear elastic fracture mechanics (LEFM) theory. Alam et al. ${ }^{21}$ and Bang et al. ${ }^{22}$ carried out parametric two-dimensional FE analyses to identify the stress intensity factors in tensile and shear bump tests for a constant-length singular crack inside the IMC layer. Although characterization of interfacial fracture of solder by employing stress intensity factors related to LEFM theory could be valuable, it should be noted that LEFM is useful when the inelastic deformation is small compared with the size of the crack (small-scale yielding). Accordingly, because of the significant viscoplastic deformation of the solder materials near the crack tip, LEFM is not a generally acceptable model. Moreover, by considering a constant crack length in the analyses of the interfacial failure of the joint, the effect of loading history is ignored.

In addition, several results have been published on damage mechanics modeling of interfacial failure of solders, mostly under fatigue loading. ${ }^{20,23,25,28}$ In most such investigations, the deformation behavior of the bulk solders was represented by simplified material models. Abdul-Baqi et al. ${ }^{20}$ and Bhate et al. ${ }^{23}$ simulated damage initiation and progression at the interface of the solder joint by utilizing the cohesive zone methodology, while the bulk material was assumed to be linear elastic. Yang et al. ${ }^{25}$ went further by considering plasticity in bulk solder. They assumed that the loading rate is very high to ignore the effects of rate-dependent viscoplasticity, which is an acceptable hypothesis. However, it should be noted that, when the strain rate is not sufficiently high, the viscoplastic deformation in solder is not generally negligible. ${ }^{4,5}$

The main scope of this paper is to shed light on the mutual effect of the viscoplastic response of bulk solder and interfacial damage of lead-free solder joints by investigating the interfacial fracture behavior at different strain rates. For this purpose, the interfacial damage evolution, consequent crack growth, and mode I fracture behavior of solder joints were characterized experimentally by developing an optimized experimental design and performing several stable interfacial fracture tests at different strain rates. An identical rate-independent cohesive zone damage model was identified, using a detailed macroscopic 3D FE model taking into account the viscoplastic behavior of the bulk solder, to best simulate the interfacial damage progression in the TDCB fracture tests. The effect of strain rate and viscoplastic deformation on the fracture mechanisms of lead-free solder joints were pointed out by analyzing the experimental and simulation results. Due to the scatter in the existing creep database of solders, the viscoplastic response of the solder joint was evaluated by performing shear tests at different strain rates, modeled by Anand constitutive relations and employed in the simulations of interfacial damage to ensure the consistency of the results.

\section{MATERIALS AND FABRICATION PROCESS}

Among the different candidates for the replacement of conventional lead solder, the near-eutectic ternary system of $\mathrm{Sn}, \mathrm{Ag}$, and $\mathrm{Cu}$, called $\mathrm{SAC}$ for short, has attracted great interest in recent years, since it satisfies essential economical and technological criteria such as low melting point, good wetting, and suitable thermomechanical properties. $^{3}$ In this study, near-eutectic SAC solder with a composition of Sn-4.0 wt.\% Ag-0.5 wt. \%Cu (SAC405, AIM solder) in the form of flux paste was utilized. As the metallurgical substrate, copper plates were employed. It is well established that the microstructures of solders and consequently the mechanical behavior of the joints are considerably sensitive to the fabrication process. ${ }^{29}$ Utilization of reflow soldering as the production method, due to its good controllability and repeatability, resulted in fabrication of joints with similar microstructures throughout this investigation. The reflow process was conducted in a Torch Co. T200C 3.8 KW programmable oven. It was shown that the use of a nonoptimal reflow profile can lead to the creation of macrovoids in solidified solder. ${ }^{17}$ To solve this problem, the micro-computed tomography $(\mu \mathrm{CT})$ technique was employed to observe the macrovoids distributed in the reflowed solder, and reflow parameters were tuned so as to produce the minimum amount of voids in the joint. The final optimized reflow profile consisted of a ramp from room 
temperature to $155^{\circ} \mathrm{C}$, dwelling for $100 \mathrm{~s}$ at this temperature, followed by a ramp to a peak temperature of $245^{\circ} \mathrm{C}$. The time above the melting temperature $\left(218^{\circ} \mathrm{C}\right)$ was $90 \mathrm{~s}$, and the samples were finally cooled in air to room temperature at a rate of approximately $0.9^{\circ} \mathrm{C} \mathrm{s}^{-1}$. The temperatures reported herein are values measured close to the solder joint.

The bulk reflowed SAC405 solder consists of $\beta$-Sn dendrites distributed in a eutectic mixture composed of $\mathrm{Ag}_{3} \mathrm{Sn}$ and $\mathrm{Cu}_{6} \mathrm{Sn}_{5}$ needle-like intermetallics dispersed in a Sn matrix. During the reflow process, molten solder reacts with the surface of the $\mathrm{Cu}$ substrate to form a metallurgical bond through the formation of $\mathrm{Sn}-\mathrm{Cu}$ intermetallic layers $\left(\mathrm{Cu}_{6} \mathrm{Sn}_{5}\right.$ and $\left.\mathrm{Cu}_{3} \mathrm{Sn}\right)^{3}$ The microstructure of a typical reflowed solder employed in this study for further mechanical testing is shown in Fig. 1. Formation of a dense and fine interfacial intermetallic layer $(\sim 3 \mu \mathrm{m})$ is the result of utilizing a well-optimized reflow profile ${ }^{9,14}$ (Fig. 1). To reveal the microstructure for optical and scanning electron microscopies, the solder specimens were degreased with ethanol,
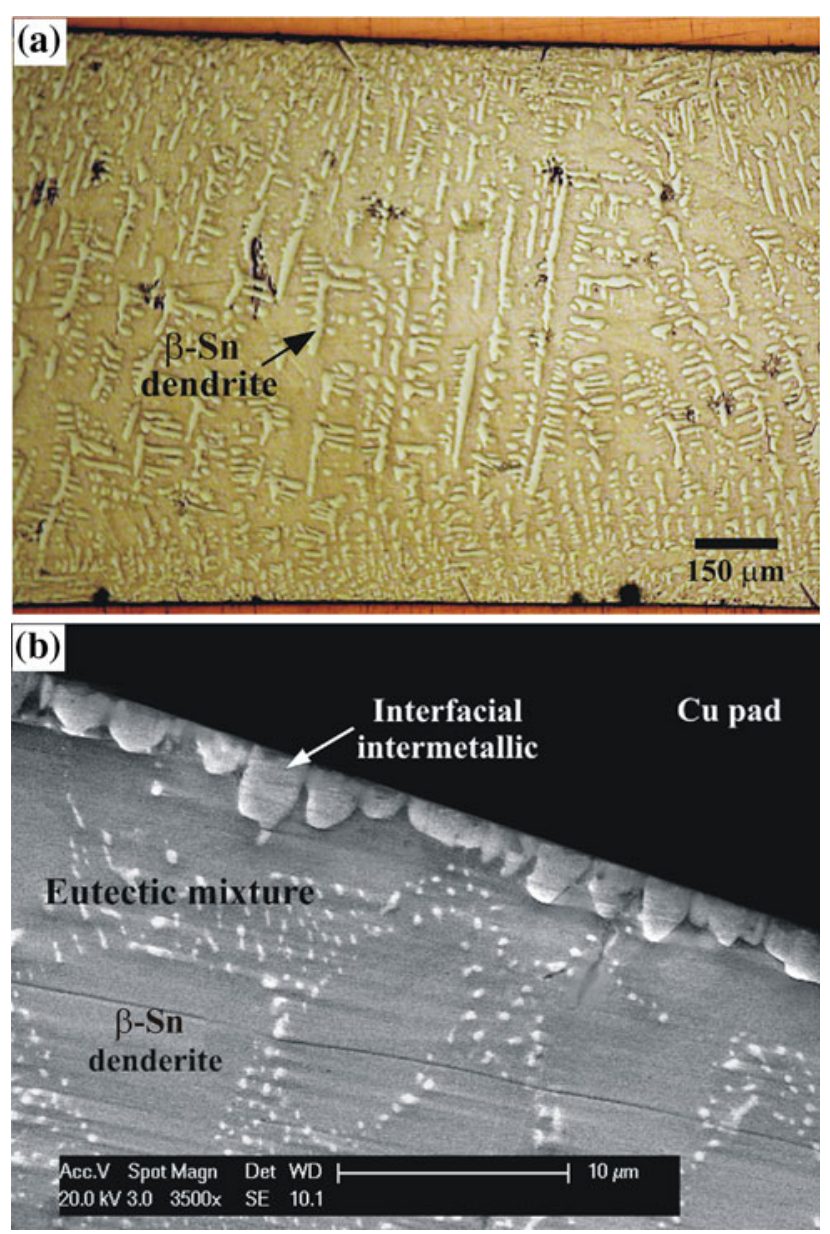

Fig. 1. (a) Optical microscopy image showing a global view of the distribution of $\beta$-Sn dendrites and (b) SEM micrograph showing the microstructures near the interface of bulk solder and Cu substrate of a SAC405/Cu solder joint. rinsed in deionized water, dried in blown air, coldmounted in epoxy resin, ground with 800,1200 , and 2400 grit $\mathrm{SiC}$ paper, and then polished to $1 \mu \mathrm{m}$. Finally, silica suspension, which is a slight etchant, was used for final polishing.

\section{EXPERIMENTAL PROCEDURES}

\section{Shear Testing}

Due to the low melting temperatures of Sn-rich lead-free solders, significant viscoplastic deformation may develop during their loading, even at room temperature. Since premature failure of the interface tends to considerably affect the results of tensile tests, ${ }^{9}$ shear testing was chosen for characterization of the viscoplastic behavior of the SAC405 solder joint. The single-lap shear specimen design as presented by Sivasubramaniam et al., ${ }^{30}$ because of its nearly uniform plastic strain distribution at the center of the joint, was utilized in this work. The geometry of the specimen and the setup used in the shear testing are shown in Fig. 2. The samples were produced as follows: surfaces of machined copper substrates were ground and cleaned in an ultrasonic-assisted cleaning machine. The $\mathrm{Cu}$ pads were tied in a fixture designed to keep the soldering gap constant. Afterward, the solder paste was poured to fill the gap and the sample was reflowed according to the profile described above. Specimens were then ground slightly to remove any excess solder. Drilling of the two lateral holes was carried out in a precisely aligned clamping fixture, ensuring that the solder was not loaded during the operation. Shear tests were performed on an Instron 5848 microtester at five different strain rates from $0.07 \% \mathrm{~s}^{-1}$ to $0.24 \% \mathrm{~s}^{-1}$ at room temperature. The digital image correlation technique (DIC) was used to measure the strain distribution in the solder joint. For this purpose, a series of high-resolution images of the joints were taken during the shear tests. The images were processed using a customdeveloped DIC software to calculate the evolution of the shear strain in the solder by tracking predefined points on the surface of the joint. Furthermore, the stress-strain behavior of the copper substrate was determined by performing tension tests on $\mathrm{Cu}$ dogbone specimens according to ASTM E8 on a MTS $809 \mathrm{axial} /$ torsional testing machine.

\section{Fracture Testing}

A stable crack growth fracture test enables the acquisition of data during damage and crack progression which are valuable for evaluation of fracture behavior and identification of an interfacial damage model. For this reason, a TDCB specimen was designed and employed in the present study for characterization of interfacial fracture of solder joints over a wide range of strain rates. Since a change in the volume of the soldering zone may influence the heat conduction during solidification 

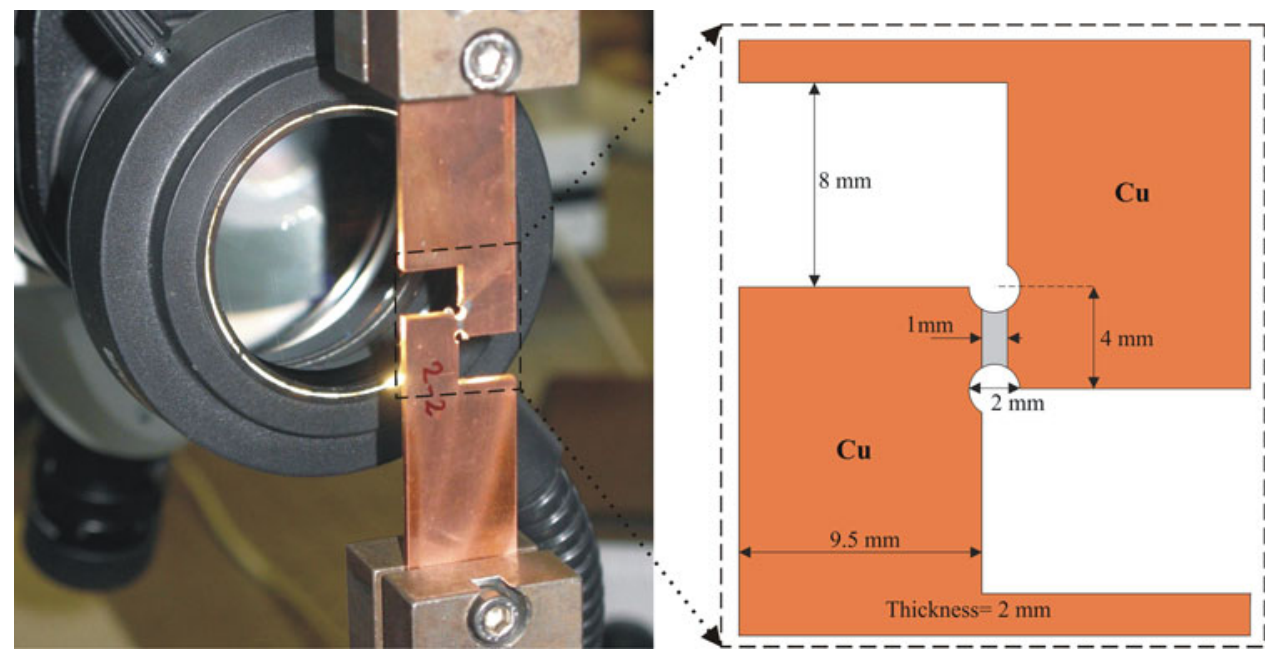

Fig. 2. Single-joint shear specimen geometry and testing setup.

and strongly affects the microstructure, the thickness of the fracture specimen should be as close as possible to that of joints applied in the electronic industry. However, the TDCB specimen geometries proposed in the literature ${ }^{31,32}$ are mainly adapted to large-dimension single-material specimens. Thus, a small TDCB joint was designed for the present application.

In a TDCB made of a linear elastic material with crack length $a$, the crack starts to propagate in mode I when the stress intensity factor $K_{\mathrm{I}}$ reaches its critical value $K_{\text {IC }}$. Hereby, the stability of the propagating crack is achieved when, for an increment of crack length $\delta a$, the stress intensity factor decreases, so that the stability criterion is $\delta K_{\mathrm{I}} /$ $\delta a<0$. In a more general case, if we also consider the plasticity of the material, part of the external work is consumed in developing plastic deformation. Consequently, in comparison with the elasticmaterial TDCB, additional energy is normally needed to grow the crack in this case, so that the crack propagation should be stable as well. Thus, it is reasonable to design a TDCB specimen based on the LEFM crack stability criterion and use it to investigate more complex material behavior. For this purpose, FE models of different TDCB geometries with elastic material behavior and different crack lengths were developed. The stress intensity factors were calculated based on the contour integral method, ${ }^{33,34}$ and the geometry of the TDCB was modified to satisfy $\delta K_{\mathrm{I}} / \delta a<0$ over a reasonably large range of crack length. ${ }^{31}$ The resulting geometry of the TDCB designed on this basis is shown in Fig. 3. The crack stability in this design was experimentally verified for the cases of a homogeneous brittle material, plexiglass, and SAC405/Cu solder joints. Since most solder joints experience mixed-mode loading in service, shear can play an important role in the development of interfacial cracks. ${ }^{24,26}$ However, comparison of the simulated stress components near the crack tip of the designed

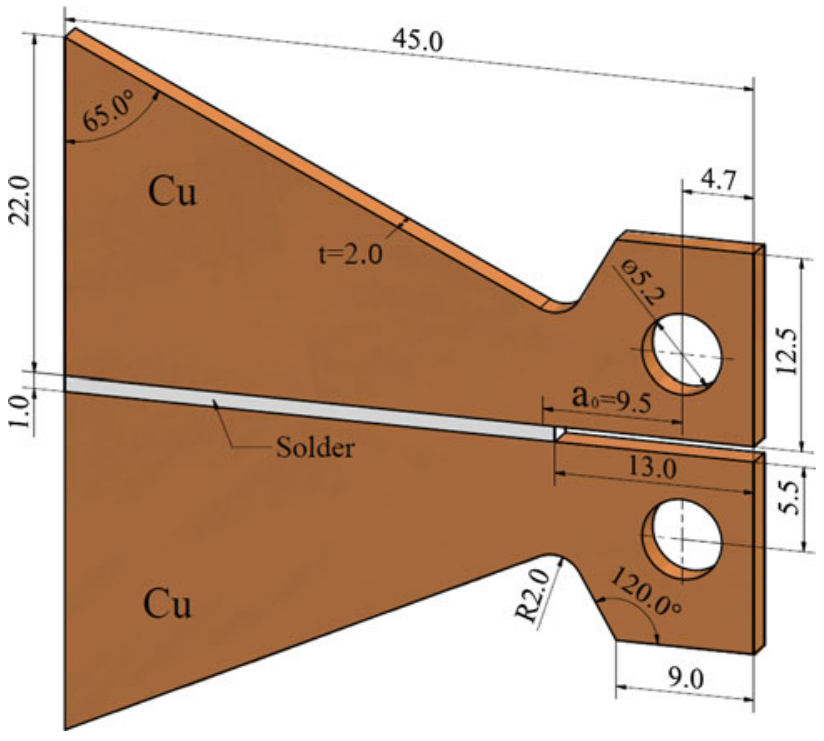

Fig. 3. Geometry of the soldered TDCB specimen designed and employed in the fracture tests.

TDCB shows that the magnitude of the local normal stress (stress component perpendicular to the crack plane) is much higher than the shear stresses. Therefore, mode I is considered as the dominant fracture mode in the TDCB design shown in Fig. 3.

For the fabrication of a soldered TDCB specimen, two halves of TDCB parts were machined out of 2 -mm-thick copper sheets. Joining surfaces were ground and polished carefully, and the $\mathrm{Cu}$ substrates were put into a solution of $0.5 \% \mathrm{HCl}$ and 99.5\% ethanol under ultrasonic vibration for $5 \mathrm{~min}$ to be cleaned. The two halves of the TDCB specimen were then aligned, leaving a $1 \mathrm{~mm}$ gap, and clamped in a specific fixture for soldering. The SAC405 solder paste was poured, and a thin Teflon foil was placed at the interface of solder and copper to create an initial crack with a length of $9.5 \mathrm{~mm}$ (Fig. 3). Soldering was then carried out according to 
the reflow profile previously described. Finally, after slight grinding of the reflowed samples, their surfaces were painted with a white spray to facilitate crack tip tracking during the fracture tests. The TDCB soldered specimens were mounted into a designed clevis grip on an Instron 5848 microtester by inserting two pins through the holes of the samples. A preload of less than $4 \mathrm{~N}$ was applied to eliminate any initial possible slip in the system. The specimens were then pulled to fracture at three different displacement rates of $0.0035 \mathrm{~mm} \mathrm{~s}^{-1}$, $0.01 \mathrm{~mm} \mathrm{~s}^{-1}$, and $0.027 \mathrm{~mm} \mathrm{~s}^{-1}$. To examine and subsequently remove any effect of the compliance of the grips and testing machine on the experimental results, DIC was employed to measure the relative displacement of the loading pins. All fracture tests were recorded with a high-resolution camera at a rate of $10 \mathrm{fps}$, used for monitoring the interfacial crack advance.

\section{EXPERIMENTAL RESULTS AND DISCUSSION}

The data in Fig. 4 show equivalent von Mises stress versus equivalent strain curves obtained from shear testing of the solder joints at five different strain rates from $0.07 \% \mathrm{~s}^{-1}$ to $0.24 \% \mathrm{~s}^{-1}$ at room temperature. The equivalent stress and strain were determined by analyzing the load and displacement obtained from DIC measurements. Each curve in Fig. 4 represents the average stress-strain response of at least three identical specimens at that strain rate. The data in Fig. 4 demonstrate the strong effect of the strain rate on the initial yield strength and hardening of the SAC405 solder joint in such a manner that the gradual increase of the imposed strain rate leads to a significant increase in the level of ultimate stress from $38 \mathrm{MPa}$ to $51 \mathrm{MPa}$.

The pin load and crack length against the corresponding displacement obtained from the fracture tests of soldered TDCB specimens at three different

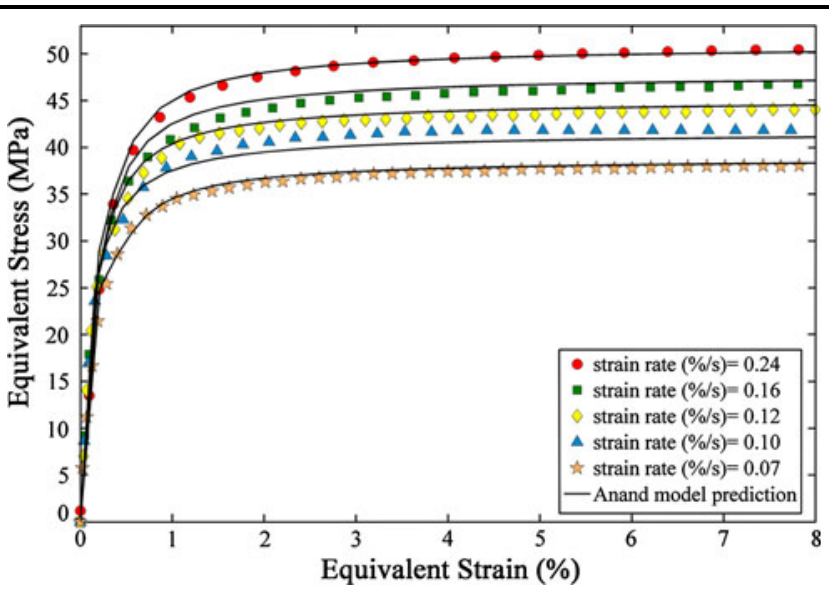

Fig. 4. Stress-strain curves obtained from shear tests of SAC405/ $\mathrm{Cu}$ solder joints at different strain rates and prediction of Anand constitutive model at room temperature. displacement rates $\left(0.0035 \mathrm{~mm} \mathrm{~s}^{-1}, 0.01 \mathrm{~mm} \mathrm{~s}^{-1}\right.$, and $0.027 \mathrm{~mm} \mathrm{~s}^{-1}$ ) are shown in Fig. 5a and b, respectively. Note that the displacement reported herein corresponds to the relative displacement of the pins measured by DIC. The solid lines in Fig. 5a, $b$ represent the average of at least three TDCB traction tests at each displacement rate, while the error bars indicate the maximum deviation of the tests from the averaged curves. As Fig. 5b shows, in the case of fracture tests at a loading rate of $0.01 \mathrm{~mm} \mathrm{~s}^{-1}$, considered as the reference speed, the crack along the interface of the solder and $\mathrm{Cu}$ started to grow after approximately $0.12 \mathrm{~mm}$ of displacement. The crack propagated in a stable manner up to a displacement of about $0.21 \mathrm{~mm}$. Thereafter, the crack growth rate increased and finally the sample suddenly broke at a displacement of approximately $0.24 \mathrm{~mm}$. In the tests performed at a displacement rate of $0.0035 \mathrm{~mm} \mathrm{~s}^{-1}$, three times slower than the reference rate, crack initiation took place after significant plastic deformation in the bulk solder at a displacement of around $0.2 \mathrm{~mm}$.
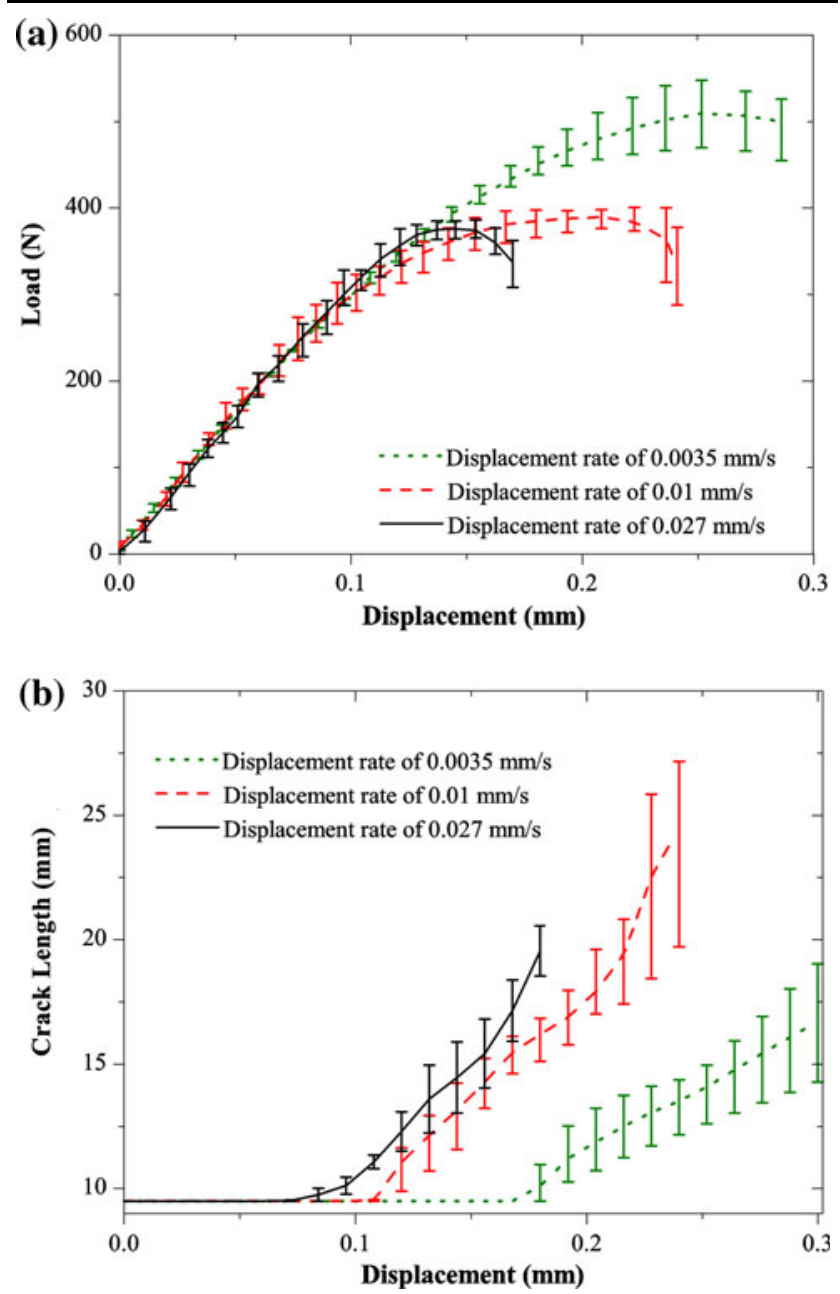

Fig. 5. (a) Load-displacement responses and (b) corresponding experimental crack length obtained from traction tests of TDCB specimens at different displacement rates. 


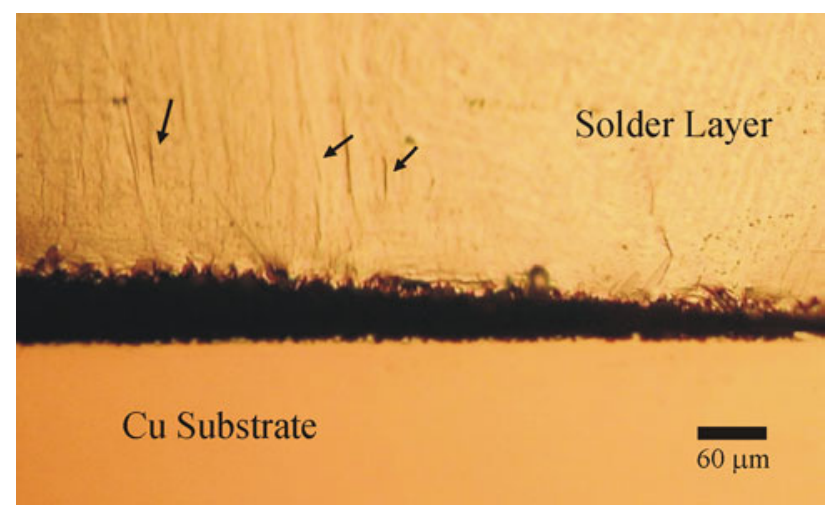

Fig. 6. Optical microscopy image of TDCB solder joint during the fracture test performed at a displacement rate of $0.0035 \mathrm{~mm} \mathrm{~s}^{-1}$, showing the development of plastic wrinkles in the bulk solder.

Then, the interfacial crack propagated slowly until around $0.28 \mathrm{~mm}$ of displacement before final fracture. In the tests at this rate, contrary to the two other rates, development of plastic deformation during the crack growth was obvious. Figure 6 shows the plastic wrinkles created at the surface of solder layer during a TDCB fracture test at a displacement rate of $0.0035 \mathrm{~mm} \mathrm{~s}^{-1}$. Alternatively, in the tests performed at a displacement rate of $0.027 \mathrm{~mm} \mathrm{~s}^{-1}$, i.e., around three times faster than the reference rate, the crack propagation started earlier, typically after around $0.07 \mathrm{~mm}$ to $0.09 \mathrm{~mm}$ of displacement. The crack grew in a stable manner, but faster than in the two previous tests, and finally the sample broke after approximately $0.18 \mathrm{~mm}$ of displacement. In brief, it was observed that the interfacial crack growth behavior in the performed TDCB fracture tests is obviously a function of the imposed displacement rate, such that with increasing displacement rate, both crack initiation and final failure occur at lower displacements (Fig. 5b). Moreover, the data in Fig. 5a demonstrate that, by increasing the displacement rate, the maximum load capacity of the joints decreases.

After failure, the specimens were carefully removed from the testing machine for fracture surface analyses. A global view of a typical fracture surface is shown in Fig. 7a. Observations and analyses of the fracture surfaces showed that generally they are composed of a mixture of facets and dimple structures (Fig. 7b). Dimples are well distributed between facets with a diameter between $5 \mu \mathrm{m}$ and $30 \mu \mathrm{m}$. The results of several qualitative and quantitative energy-dispersive $\mathrm{x}$-ray spectroscopy (EDX) analyses conducted on the fracture surfaces showed that the facets [arrow (a) in Fig. $7 \mathrm{~b}$ ] have the same composition as the interfacial intermetallic layer, i.e., $\mathrm{Cu}_{6} \mathrm{Sn}_{5}$ intermetallic. However, the dimples are mainly composed of $\mathrm{Sn}$ matrix [arrow (b) in Fig. 7b] containing needleshape microstructures [arrow (c) in Fig. 7b] with similar compositions to the needles in the eutectic mixture $\left(\mathrm{Ag}_{3} \mathrm{Sn}\right.$ and $\mathrm{Cu}_{6} \mathrm{Sn}_{5}$ needles in Fig. $\left.1 \mathrm{~b}\right)$.
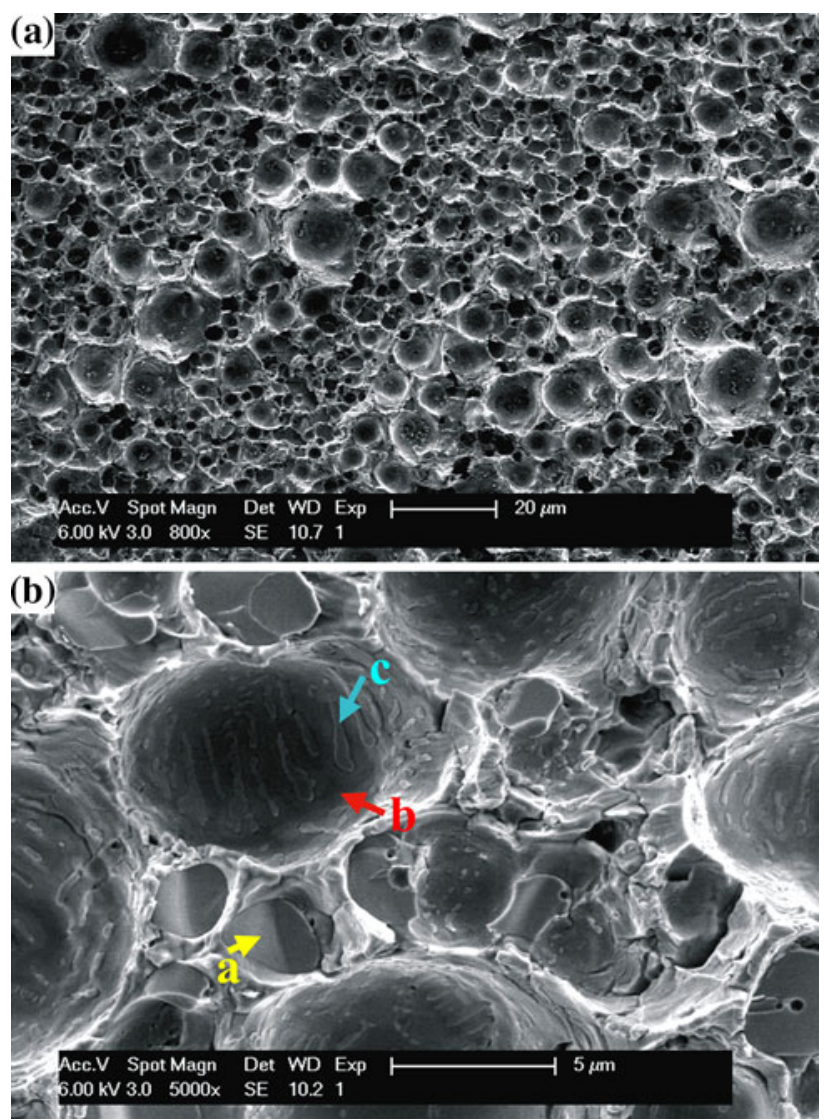

Fig. 7. (a) Global view and (b) higher-magnification SEM micrograph of fracture surface, showing mixture of dimpled surface (fracture inside the solder close to IMC) and brittle fracture in IMC.

Therefore, it is concluded that, in the fracture of SAC405/Cu soldered TDCB specimens, the crack typically grows near the interface of IMC and bulk solder and may go inside the IMC or bulk solder during its propagation. Since IMC and bulk solder display brittle and ductile features, respectively, the surface is a mixture of dimples and flat faces. This is in agreement with the observation of Hayes et al. ${ }^{14}$

\section{MODELING}

\section{Constitutive Modeling of Viscoplastic Behavior}

Anand model ${ }^{35}$ was employed to characterize the constitutive inelastic response of SAC405 solder joints under shear at room temperature. The original Anand model and some extensions were previously used to represent the viscoplastic deformation behavior of different types of lead-free solders in tension. ${ }^{13,36,37}$ Anand viscoplastic model specifically is a set of constitutive equations in which timedependent creep and rate-independent plastic strain can be modeled in a unified formulation. The model employs a single scalar $s$ as an internal variable to represent hardening. The equivalent von Mises stress $\sigma$ can be expressed as a function of $s$ by 


$$
\sigma=c s, \quad c<1
$$

where $c$ is a function of strain rate and temperature expressed as follows:

$$
c=\frac{1}{\xi} \sinh ^{-1}\left\{\left[\frac{\dot{\varepsilon}_{\mathrm{p}}}{A} \exp \left(\frac{Q}{R T}\right)\right]^{m}\right\}
$$

where $\dot{\varepsilon}_{\mathrm{p}}$ is the plastic strain rate, $\xi$ is a material constant, $A$ is an exponential factor, $m$ is the strain rate sensitivity, $Q$ is the activation energy, $R$ is the universal gas constant, and $T$ is absolute temperature. ${ }^{13,36,37}$ Since we focused on constant room-temperature behavior of solder, Eq. (2-1) can be simplified by defining $A^{\prime}=1 / A \exp (Q / R T)=$ cte as follows:

$$
c=\frac{1}{\xi} \sinh ^{-1}\left\{\left[A^{\prime} \dot{\varepsilon}_{\mathrm{p}}\right]^{m}\right\}
$$

In Anand model, the evolution equation is

$$
\dot{s}=\left\{h_{0}\left|1-\frac{s}{s^{*}}\right|^{a} \operatorname{sign}\left(1-\frac{s}{s^{*}}\right)\right\} \dot{\varepsilon}_{\mathrm{p}},
$$

where $a$ and $h_{0}$ are material parameters representing hardening, and $s^{*}$ is the saturation value of $s$ at a given strain rate:

$$
s^{*}=\hat{s}\left[A^{\prime} \dot{\varepsilon}_{\mathrm{p}}\right]^{n} .
$$

Detailed descriptions of the model can be found in the literature. ${ }^{13,36,37}$ Here, $A^{\prime}, \hat{s}, h_{0}, s_{0}, m, n, a$, and $\xi$ are material parameters which should be determined. A specific direct fitting approach ${ }^{36,37}$ was applied for the identification of the constitutive parameters of the model based on the response of SAC405 solder joints under shear shown in Fig. 4. Table I presents the material parameters identified for lead-free SAC405, and the solid lines in Fig. 4 show the predicted responses for the solder joints. The identified model was used in the simulation of fracture tests.

\section{Cohesive Modeling of Interfacial Damage}

The cohesive zone methodology is a convenient numerical tool applicable to modeling of crack initiation and propagation at the interface of similar or dissimilar materials such as adhesive joints and layers in composites. ${ }^{38,39}$ In this method, the pertinent material properties, such as fracture energy and fracture strength, are taken into account in a damage mechanics model which represents the evolution of the interfacial stresses as a function of

the opening displacements and accumulated damage. Accordingly, crack initiation and propagation appear in the solution of the problem without any additional criteria. ${ }^{20,38,39}$

In the present study, a bilinear normal tractionseparation model was employed for the simulation of interfacial damage initiation and progression in the TDCB fracture tests, because of its simplicity and robustness (Fig. 8). In this model, by increasing the cohesive separation, the cohesive traction initially increases linearly and elastically with a slope of $K$. Once the traction in the cohesive zone reaches the value of $T_{\mathrm{D} 0}$, damage initiates. Upon further loading, evolution of damage from initiation to complete failure is controlled by the energy dissipated as a result of the damage process, i.e., the cohesive fracture energy which is equal to the area under the traction-separation curve of Fig. 8, denoted as $\Gamma$. Since mode $I$ is the dominant fracture mode in the TDCB design, development of damage due to shear was ignored in the employed cohesive model. However, in a general case, the shearinduced damage should be taken into account in the cohesive modeling of interfacial failure of solders by considering shear traction-separation behavior.

To apply the cohesive zone model in the simulation of the interfacial damage growth, the model parameters, i.e., the initial stiffness of the cohesive zone $K$, the damage initiation traction $T_{\mathrm{Do}}$, and the cohesive fracture energy $\Gamma$, should be determined. The value of $K$ should be chosen sufficiently high in order to minimize its effect on the overall compliance of the model. ${ }^{20,39}$

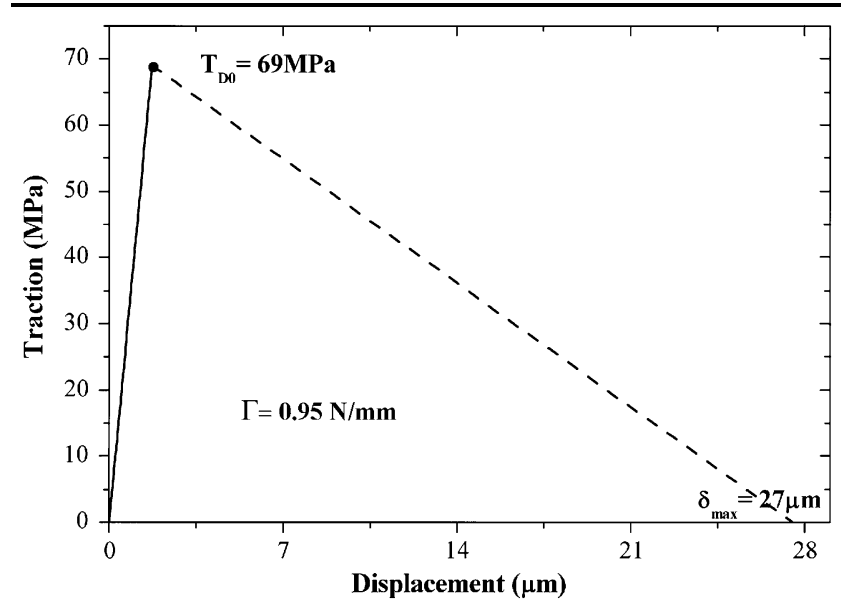

\begin{tabular}{|c|c|c|c|c|c|c|c|c|}
\hline Material Parameters: & $A^{\prime}(\mathrm{s})$ & $\hat{s}(\mathrm{MPa})$ & $h_{0}(\mathrm{MPa})$ & $s_{0}(\mathrm{MPa})$ & $m$ & $n$ & $a$ & $\xi$ \\
\hline Value: & 83,923 & 45.84 & 76,600 & 31.98 & 0.888 & 0.042 & 2.2 & 6 \\
\hline
\end{tabular}

Fig. 8. Cohesive traction-separation curve of the employed cohesive zone constitutive model.

Table I. Material parameters of Anand constitutive model determined based on the viscoplastic response of SAC405/Cu solder joint in shear 
Regarding $T_{\mathrm{D} 0}$ and $\Gamma$, these were identified numerically in such a way as to simulate the interfacial failure of solder as observed in the TDCB fracture tests in detail. For this purpose, a threedimensional FE model was constructed in Simulia Abaqus Standard v6.8 based on the actual geometry of the TDCB joint. To reflect the viscoplastic behavior of the lead-free solder, Anand constitutive model with the material parameters presented in Table I was employed in the FE model. The elastoplastic behavior of the copper substrate obtained from the tensile test was also implemented directly in the FE model as a tabular yield stressplastic strain relationship (isotropic hardening). A layer of cohesive elements with a thickness of $10 \mu \mathrm{m}$ and unknown cohesive parameters was implemented at the interface of the solder and $\mathrm{Cu}$ substrates. This layer represents physically the interphase region where failure takes place, namely the IMC layer and small surrounding region of the bulk solder. The cohesive layer was finely meshed by linear hexagonal eight-node $3 \mathrm{D}$ cohesive elements with dimensions of $10 \mu \mathrm{m} \times 12 \mu \mathrm{m} \times 12 \mu \mathrm{m}$. The different regions of the model (substrates, solder, and cohesive layer) were linked together using kinematic tie constraints. A global and zoomed view of the FE model are shown in Fig. 9. As can be seen, relatively small elements (size $\sim 60 \mu \mathrm{m}$ ) were utilized to discretize the solder part and copper pad near the cohesive layer to ensure convergence of the local fields. Overall, the detailed 3D FE model consisted of 574,795 nodes and 354,205 elements for a problem size of approximately 1,060,000 degrees of freedom. It should be noted that the employed cohesive model is chosen to be rate independent and the only rate dependency in the FE model comes from the viscoplastic behavior of the solder. To identify the $T_{\mathrm{D} 0}$ and $\Gamma$ values of the model, several FE simulations with identical cohesive zone parameters were performed for each loading rate used in the experiments. The simulation results were then compared with the corresponding experimental data of the TDCB tests. The cohesive parameters, $T_{\mathrm{D} 0}$ and $\Gamma$, were tuned in an identification loop to best approximate the experimental load-displacement curves of Fig. 5a as well as the corresponding interfacial crack lengths of Fig. 5b. The values of $T_{\mathrm{D} 0}$ and $\Gamma$ were finally identified to be $69 \mathrm{MPa}$ and $0.95 \mathrm{~N} \mathrm{~mm}^{-1}$, respectively. The solid lines in Fig. 10a and b, respectively, show the load and crack length versus displacement obtained from FE simulations of TDCB tests with the identified cohesive zone parameters, while the error bars represent the range of the experimental results as shown in Fig. 5. Comparison of the experimental and numerical data in Fig. 10 indicates that the identified cohesive and viscoplastic constitutive models are appropriate to simulate the TDCB fracture tests. For a better understanding of the data in Fig. 10b, images of a TDCB specimen (zoomed to the solder area) during a fracture test after $0.09 \mathrm{~mm}, 0.12 \mathrm{~mm}, 0.15 \mathrm{~mm}$, and $0.17 \mathrm{~mm}$ of displacement at a loading rate of $0.027 \mathrm{~mm} \mathrm{~s}^{-1}$ are shown in Fig. 11. Note that continuous crack growth is observed at the interface of the solder and copper in Fig. 11a-d. The colored bands superimposed on each image of Fig. 11 represent contour plots of the damage parameter $D$ in the plane of cohesive layer at the same displacements $(0.09 \mathrm{~mm}$, $0.12 \mathrm{~mm}, 0.15 \mathrm{~mm}$, and $0.17 \mathrm{~mm}$ ) obtained from the simulation of TDCB tests at the rate of $0.027 \mathrm{~mm} \mathrm{~s}^{-1}$. The red and blue contours represent fully damaged (cracked zone) and undamaged areas, respectively. Comparison of the images and corresponding contour plots shows that nearly the same crack length was obtained in the simulation and the test results. It should be mentioned that FE simulation of crack growth beyond crack length of about $17 \mathrm{~mm}$ was not possible due to a convergence problem arising from crack instability.

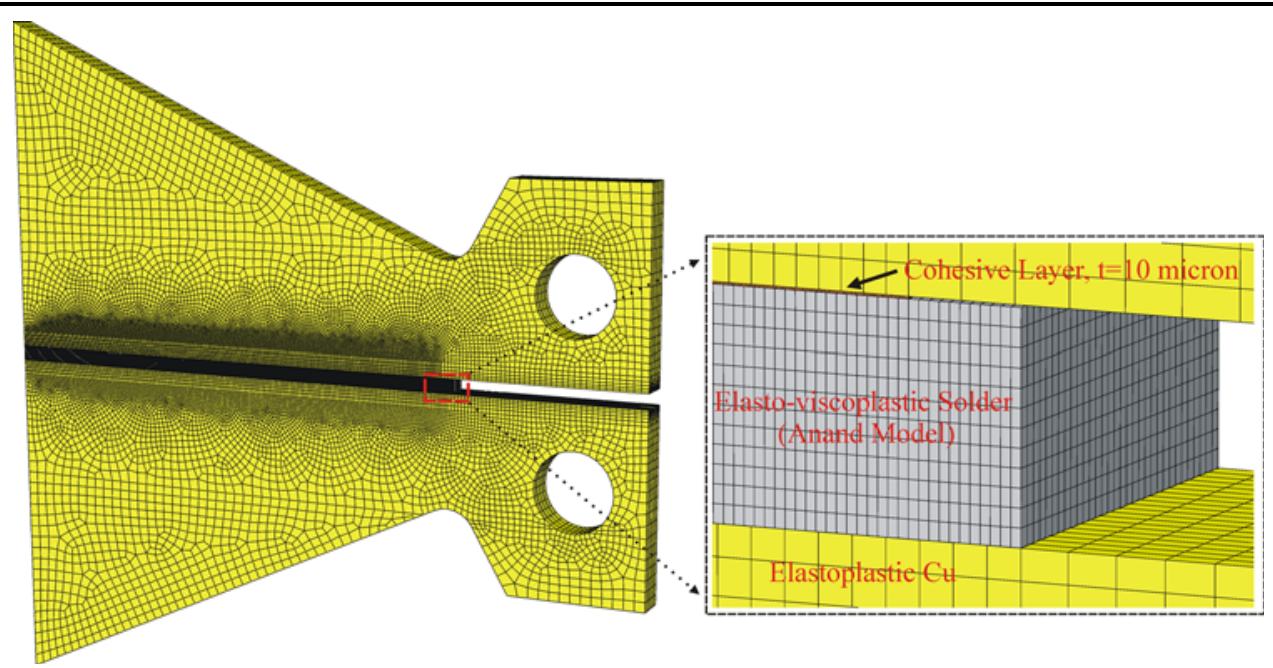

Fig. 9. Global and zoomed view of the 3D FE model of TDCB showing implemented cohesive layer and mesh quality around solder area. 

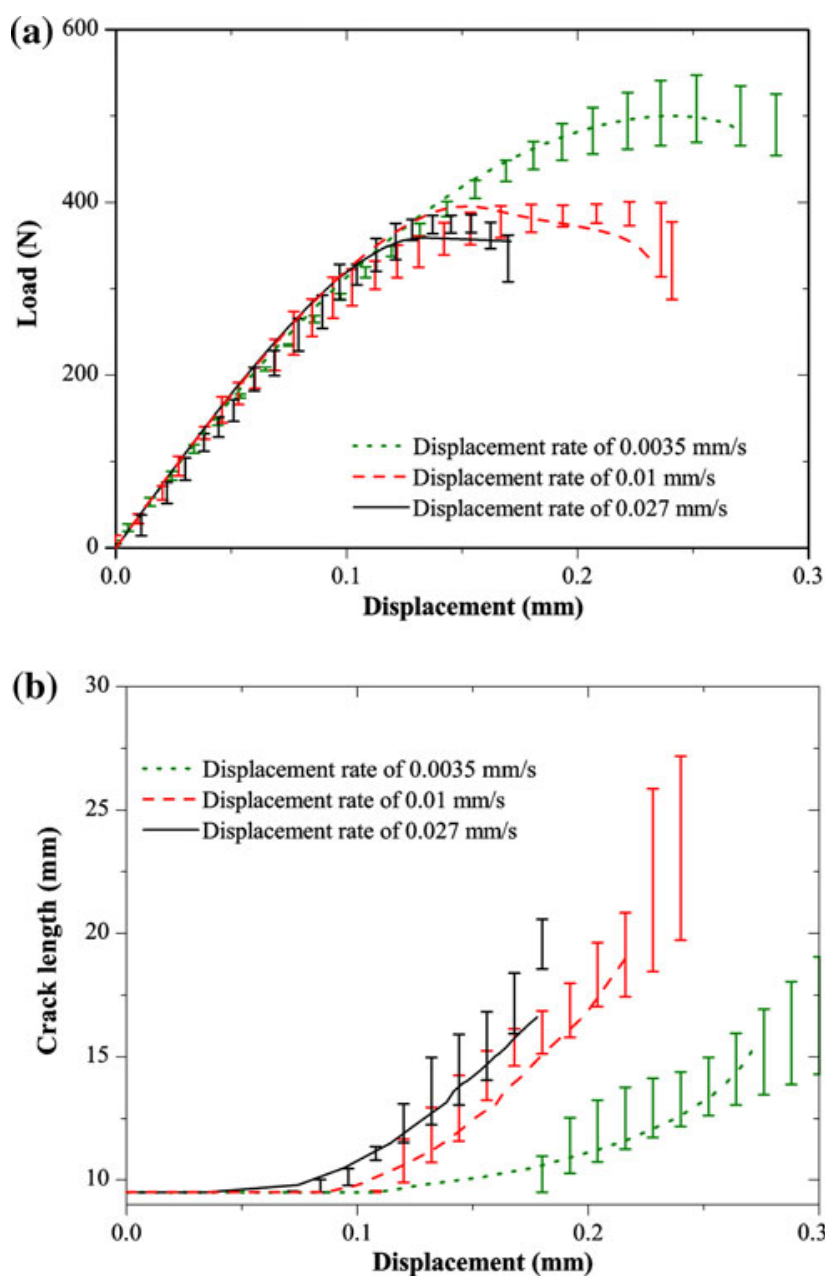

Fig. 10. Comparison of (a) load-displacement responses and (b) crack propagation behavior obtained from FE simulations and traction tests of TDCB specimens at different displacement rates.

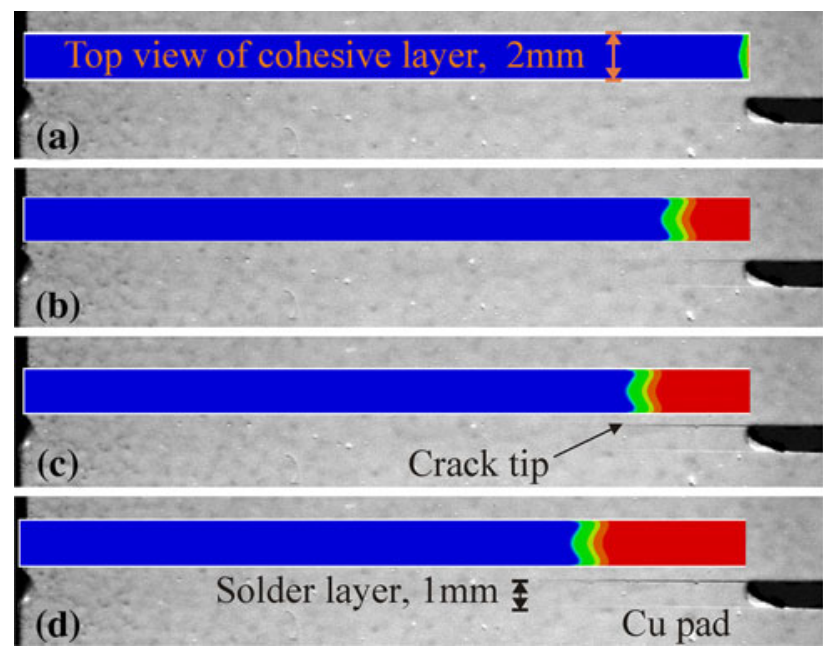

Fig. 11. Comparison of experimental crack length and numerical damage distribution in the cohesive layer after opening displacement of (a) $0.09 \mathrm{~mm}$, (b) $0.12 \mathrm{~mm}$, (c) $0.15 \mathrm{~mm}$, and (d) $0.17 \mathrm{~mm}$ at rate of $0.027 \mathrm{~mm} \mathrm{~s}^{-1}$.

\section{SIMULATION RESULTS AND DISCUSSION}

The data in Fig. 4 demonstrate that the original Anand model can be reliably utilized to simulate the viscoplastic response of SAC405 solder joints in shear at room temperature in the considered strain rate range. Moreover, the good coherence between the results of fracture tests and $\mathrm{FE}$ simulations, shown in Figs. 10 and 11, highlights the robustness of the identified cohesive zone model for the simulation of interfacial crack growth in the solder joint for the range of loading rates considered herein.

According to the identified cohesive zone model, the maximum separation in the cohesive layer causing complete damage of the interfacial layer, $\delta_{\max }$ in Fig. 8, is $27 \mu \mathrm{m}$. Using optical microscopy techniques, the depths of the dimples at both fracture surfaces of the TDCB traction specimens were found in the range of $10 \mu \mathrm{m}$ to $24 \mu \mathrm{m}$, which indicates that a $20 \mu \mathrm{m}$ to $48 \mu \mathrm{m}$ separation might happen before interfacial fracture of the joint. The close values of the separation in the cohesive layer $\delta_{\max }$ and the experimental critical separation distance could be seen as physical proof of the cohesive model and the parameters identified in the present work.

The external work applied on the TDCB specimen during fracture tests is distributed into four mechanisms: it can be stored as elastic strain energy in the specimen, dissipated in the solder due to viscoplasticity, dissipated in the copper substrates by developing plasticity, and/or used for interfacial damage progression. Employing the FE model developed herein, the allotment of the external work during the TDCB fracture tests was computed. Figure 12 presents the simulated energy balance in the TDCB after a displacement of $0.17 \mathrm{~mm}$ at different displacement rates. According to the data in Fig. 12, almost $70 \%$ of the external work is preserved as elastic strain energy for all three different testing rates. In the case of the lowest displacement rate $\left(0.0035 \mathrm{~mm} \mathrm{~s}^{-1}\right), 57 \%$ of the remaining energy is consumed by viscoplasticity and only $6 \%$ is dissipated into interfacial damage. At the intermediate displacement rate $\left(0.01 \mathrm{~mm} \mathrm{~s}^{-1}\right), 32 \%$ and $47 \%$ of dissipation corresponds to viscoplasticity and damage progression, respectively. For the case of the highest displacement rate $\left(0.027 \mathrm{~mm} \mathrm{~s}^{-1}\right)$, the corresponding energies are $19 \%$ and $68 \%$ of the total dissipated energy. Accordingly, the main energy dissipation mechanism at the lowest and the highest displacement rates are viscoplastic deformation of the bulk solder and interfacial damage progression, respectively. Namely, with increasing testing rate, the role of interfacial damage and the tendency for interfacial crack growth increase significantly.

The distributions of the equivalent inelastic strains in the solder joint after $0.15 \mathrm{~mm}$ of displacement at loading rates of $0.0035 \mathrm{~mm} \mathrm{~s}^{-1}$ and $0.027 \mathrm{~mm} \mathrm{~s}^{-1}$ are shown in Fig. 13, showing that, for the case of loading at the lower rate, significant inelastic deformation developed in the middle of the 


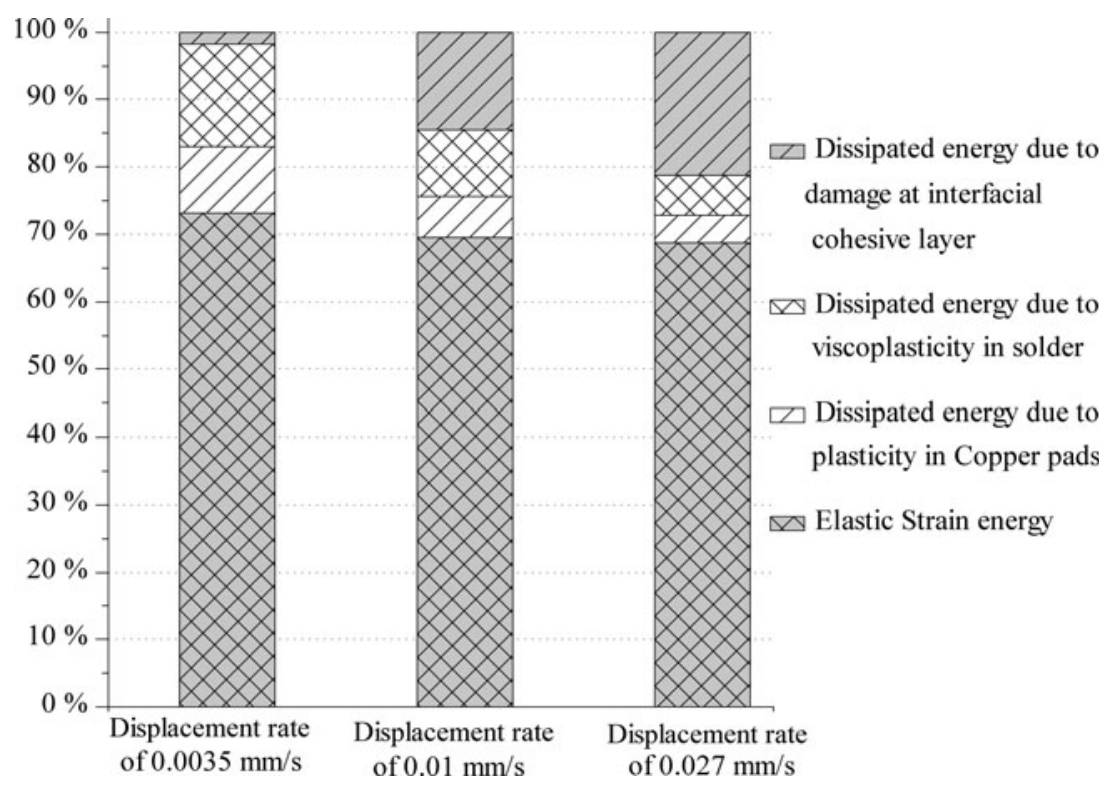

Fig. 12. External energy allotment after opening displacement of $0.17 \mathrm{~mm}$ at different displacement rates.

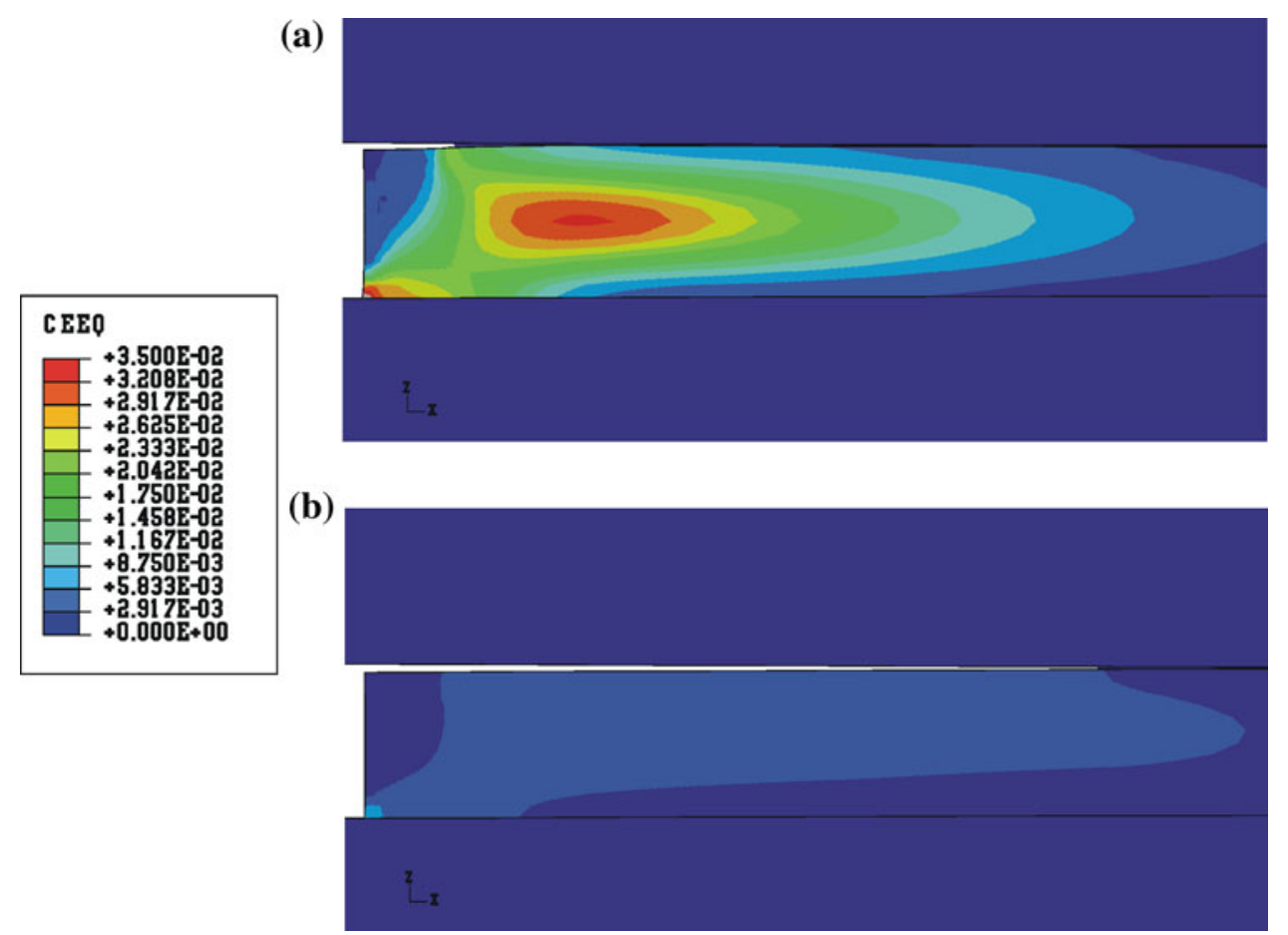

Fig. 13. Distribution of equivalent inelastic strain in the solder after $0.15 \mathrm{~mm}$ of displacement at loading rates of (a) $0.0035 \mathrm{~mm} \mathrm{~s}^{-1}$ and (b) $0.027 \mathrm{~mm} \mathrm{~s}^{-1}$.

solder layer, so that less stress was transmitted to the interface and accordingly less traction was generated in the cohesive layer. However, at the loading rate of $0.027 \mathrm{~mm} \mathrm{~s}^{-1}$, the inelastic deformation developed in the solder layer is obviously much less, which led to the growth of the interfacial crack as shown in Fig. 13.
As established experimentally, with increasing displacement rate, crack initiation occurs at lower displacement levels and the maximum load capability of the joints decreases. Indeed, the reason for this latter decrease is a gradual transition of the active failure mode from viscoplastic deformation of the bulk solder to interfacial cracking as the loading 
rate increases, as indicated in Figs. 12 and 13. Phenomenologically, at low strain rate, the normal stresses generated at the interfaces are limited by the significant creep relaxation developed in the bulk solder and thus are not sufficiently high to initiate an interfacial crack. In a sense, at low strain rate, the viscoplastic solder acts as a stress concentration damper for the interface. On the contrary, at high loading rate, the creep strain rate of the bulk solder becomes negligible compared with the applied strain rate and thus the stresses developed at the interfaces become significantly higher, which in turn promotes the development of the interfacial failure.

According to the results of experiments and simulations, considerable amounts of both interfacial damage and viscoplastic deformation were developed during the loading at a displacement rate of $0.01 \mathrm{~mm} \mathrm{~s}^{-1}$. Therefore, compared with the responses at the two extreme loading rates used herein, a value around the intermediate displacement rate, $0.01 \mathrm{~mm} \mathrm{~s}^{-1}$, which corresponds to an equivalent strain rate of approximately $0.4 \% \mathrm{~s}^{-1}$ in the solder, could be considered as the failure mode transition rate for the system studied here. The data in Fig. 13 along with Fig. 6 show that the amount of viscoplasticity developed during the fracture tests of the solders is generally not negligible and must be accounted for in analyses of the interfacial damage of solder joints. In this regard, LEFM is generally not an appropriate theoretical framework to investigate interfacial crack propagation in lead-free solders (with the exception of high-speed or impact-like loading at low temperature). Furthermore, the results of the present study help to better understand the mechanics of the interfacial damage progression and failure observed in the tensile tests of lead-free solder joints reported by Frear and Vianco. ${ }^{9}$

\section{CONCLUSIONS}

In this study, interfacial fracture of lead-free solder joints was experimentally characterized by performing stable fracture tests at different displacement rates and modeled using a cohesive zone approach considering at the same time the viscoplastic behavior of bulk solder in the analyses employing an identified Anand viscoplastic model. The results of this research led to the following conclusions:

- The main energy dissipation mechanism at the lower loading rate is viscoplastic deformation of bulk solder, while, with increasing strain rate, the portion of energy dissipated due to interfacial damage and the tendency to develop interfacial fracture increase, which causes a decrease in the maximum load capability of the joint. The failure mode transition loading rate for the system studied here was estimated as around $0.01 \mathrm{~mm} \mathrm{~s}^{-1}$, which corresponded to an equivalent strain rate of $0.4 \% \mathrm{~s}^{-1}$ in the solder.

- The mutual effect of the viscoplastic response of the bulk solder and the interfacial damage progression is generally not negligible, which confirms the necessity to consider both processes in mechanical analyses of solder joints. Accordingly, characterization of interfacial fracture in solder joints ignoring viscoplasticity of the solder layer and, conversely, analyses of deformation in solder joints ignoring the influence of the possible interfacial damage may lead to significant errors. This also implies that use of LEFM for characterization of the fracture energy of solder joints should be considered with great caution.

- For the range of strain rates considered herein, the interfacial fracture of the solder joint could be characterized by a rate-independent model even though the solder joint failure is a rate-dependent process.

\section{ACKNOWLEDGEMENTS}

This research work has been fully supported by the Swiss Secretariat for Education and Research (SER Grant C08.0030) and was conducted in the framework of the European COST action MP 0602 HISOLD: Advanced Solder Materials for High Temperature Application.

\section{REFERENCES}

1. K.J. Puttlitz and K.A. Stalter, Handbook of Lead-Free Solder Technology for Microelectronic Assemblies (New York: CRC, 2004).

2. M. Abtew and G. Selvaduray, Mat. Sci. Eng. R-Rep. 27, 95 (2000).

3. I.E. Anderson, J. Mater. Sci. Mater. Electron. 18, 55 (2007)

4. S. Wiese and K.J. Wolter, Microelectron. Reliab. 47, 223 (2007).

5. X. Long, I. Dutta, V. Sarihan, and D.R. Frear, J. Electron. Mater. 37, 189 (2008)

6. M.A. Matin, J.G.A. Theeven, W.P. Vellinga, and M.G.D. Geers, Microelectron. Reliab. 47, 1262 (2007).

7. J. Cugnoni, J. Botsis, V. Sivasubramaniam, and J. JanczakRusch, Fatigue Fract. Eng. Mater. Struct. 30, 387 (2007).

8. Y.T. Chin, P.K. Lam, H.K. Yow, and T.Y. Tou, Microelectron. Reliab. 48, 1079 (2008).

9. D.R. Frear and P.T. Vianco, Metall. Mater. Trans. A 25, 1509 (1994)

10. M. Kerr and N. Chawla, JOM-J. Min. Met. Mater. Soc. 56, 50 (2004).

11. J.H.L. Pang, B.S. Xiong, C.C. Neo, X.R. Zhang, and T.H. Low, Electronic Components and Technology Conference, 2003, p. 673.

12. R.S. Sidhu, X. Deng, and N. Chawla, Metall. Mater. Trans. A 39, 349 (2008).

13. G.Z. Wang, Z.N. Cheng, K. Becker, and J. Wilde, J. Electron. Packaging 123, 247 (2001).

14. S.M. Hayes, N. Chawla, and D.R. Frear, Microelectron. Reliab. 49, 269 (2009).

15. C.K. Shin, Y.J. Baik, and J.Y. Huh, J. Electron. Mater. 30, 1323 (2001).

16. V. Sivasubramaniam, N.S. Bosco, J. Janczak-Rusch, J. Cugnoni, and J. Botsis, J. Electron. Mater. 37, 1598 (2008).

17. M.A. Dudek, L. Hunter, S. Kranz, J.J. Williams, S.H. Lau, and N. Chawla, Mater. Charact. 61, 433 (2010).

18. J.C. Suhling, H.S. Gale, R.W. Johnson, M.N. Islam, T. Shete, P. Lall, M.J. Bozack, J.L. Evans, P. Seto, T. Gupta, 
and J.R. Thompson, Solder. Surf. Mt. Technol. 16, 77 (2004).

19. J.J. Sundelin, S.T. Nurmi, T.K. Lepisto, and E.O. Ristolainen, Mater. Sci. Eng. A 420, 55 (2006).

20. A. Abdul-Baqi, P.J.G. Schreurs, and M.G.D. Geers, Int. J. Solids Struct. 42, 927 (2005).

21. M.O. Alam, H. Lu, C. Bailey, and Y.C. Chan, Comp. Mater. Sci. 45, 576 (2009).

22. W.H. Bang, M.W. Moon, C.U. Kim, S.H. Kang, J.P. Jung, and K.H. Oh, J. Electron. Mater. 37, 417 (2008).

23. D. Bhate, D. Chan, G. Subbarayan, and L. Nguyen, J. Electron. Packag. 130, 21003 (2008).

24. P. Kumar, I. Dutta, V. Sarihan, D.R. Frear, and M. Renavikar, 11th Conference on Thermal and Thermomechanical Phenomena in Electronic Systems, ITHERM 2008, 2008, p. 660.

25. Q.D. Yang, D.J. Shim, and S.M. Spearing, Microelectron. Eng. 75, 85 (2004).

26. S.P.V. Nadimpalli and J.K. Spelt, Eng. Fract. Mech. 78, 317 (2011).

27. A.F. Skipor, S.V. Harren, and J. Botsis, Eng. Fract. Mech. 52,647 (1995).
28. P. Towashiraporn, G. Subbarayan, and C.S. Desai, Int. J. Solids Struct. 42, 4468 (2005).

29. W. Yang, L.E. Felton, and R.W. Messler, J. Electron. Mater. 24, 1465 (1995).

30. V. Sivasubramaniam, M. Galli, J. Cugnoni, J. JanczakRusch, and J. Botsis, J. Electron. Mater. 38, 2122 (2009).

31. W. Beres, K.K. Koul, and R. Thamburaj, J. Test. Eval. 25, 536 (1997).

32. J.P. Gallagher, Eng. Fract. Mech. 3, 27 (1971).

33. T.L. Anderson, Fracture Mechanics: Fundamentals and Applications, 2nd ed. (New York: CRC, 1995).

34. C.F. Shih, B. Moran, and T. Nakamura, Int. J. Fracture 30, 79 (1986).

35. S.B. Brown, K.H. Kim, and L. Anand, Int. J. Plast. 5, 95 (1989).

36. N. Bai, X. Chen, and H. Gao, Mater. Des. 30, 122 (2009).

37. X. Chen, G. Chen, and M. Sakane, IEEE Trans. Compon. Pack. Technol. 28, 111 (2005).

38. J.L. Chaboche, F. Feyel, and Y. Monerie, Int. J. Solids Struct. 38, 3127 (2001).

39. X.P. Xu and A. Needleman, Model. Simul. Mater. Sci. 1, 111 (1993). 